PROCEEDINGS OF THE

AMERICAN MATHEMATICAL SOCIETY

Volume 128, Number 6, Pages 1793-1797

S 0002-9939(99)05387-3

Article electronically published on October 29, 1999

\title{
ON CONDITIONS FOR POLYCONVEXITY
}

\author{
JAN KRISTENSEN
}

(Communicated by Steven R. Bell)

\begin{abstract}
We give an example of a smooth function $f: \mathbb{R}^{2 \times 2} \rightarrow \mathbb{R}$, which is not polyconvex and which has the property that its restriction to any ball $B \subset \mathbb{R}^{2 \times 2}$ of radius one can be extended to a smooth polyconvex function $f_{B}: \mathbb{R}^{2 \times 2} \rightarrow \mathbb{R}$. In particular, it implies that there exists no 'local condition' which is necessary and sufficient for polyconvexity of functions $g: \mathbb{R}^{n \times m} \rightarrow \mathbb{R}$, where $n, m \geq 2$. We also briefly discuss connections with quasiconvexity.
\end{abstract}

The class of polyconvex functions was introduced by Ball in [3] following earlier work by Morrey [9]. We recall that a function $f: \mathbb{R}^{n \times m} \rightarrow \mathbb{R}$ is polyconvex if $f(X)$ can be written as a convex function of the minors of $X$. For example, if $m=n=2$, then $f$ is polyconvex if there exists a convex function $F: \mathbb{R}^{2 \times 2} \times \mathbb{R} \rightarrow \mathbb{R}$ such that $f(X)=F(X, \operatorname{det} X)(\operatorname{det} X$ denotes the determinant of the matrix $X)$. The main reason for the interest in polyconvexity stems from the fact that, at present, it is the only known tractable condition which is reasonably flexible (e.g., it is compatible with natural requirements for stored energy functions in non-linear elasticity) and which implies quasiconvexity. The notion of quasiconvexity is due to Morrey 9 and is the natural substitute for convexity in the multi-dimensional calculus of variations. The condition of polyconvexity is strictly stronger than the condition of quasiconvexity (see, e.g., [1]). We refer to the monograph of Dacorogna [4] and the lecture notes by Müller [10] for the definitions and an introduction to the various convexity notions studied in the multi-dimensional calculus of variations.

The principal result of this paper is contained in the following theorem.

Theorem 1. Assume that $n, m \geq 2$. There exists a non-polyconvex smooth function $f: \mathbb{R}^{n \times m} \rightarrow \mathbb{R}$, such that its restriction to any ball $B \subset \mathbb{R}^{n \times m}$ of radius one can be extended to a smooth polyconvex function $f_{B}: \mathbb{R}^{n \times m} \rightarrow \mathbb{R}$. If $n \geq 3, m \geq 2$, then $f$ can be taken to be non-quasiconvex.

Remarks. (1) For $m \geq 2, n \geq 6$ Šverák (see [10]) has given an example of a nonquasiconvex smooth function $f: \mathbb{R}^{n \times m} \rightarrow \mathbb{R}$, which has the property that it agrees with polyconvex functions on balls of radius 1. (2) The result stated in Theorem 1 was announced in 5 .

As an immediate consequence we deduce that there exists no 'local condition', which is equivalent to polyconvexity. To formulate this statement precisely we

Received by the editors July 29, 1998.

1991 Mathematics Subject Classification. Primary 49J10, 49J45.

Key words and phrases. Polyconvexity, quasiconvexity, rank-1 convexity.

Supported by the Danish Natural Science Research Council through grant no. 9501304.

(C)2000 American Mathematical Society 
introduce the following notation. Denote by $\mathcal{C}^{\infty}=\mathcal{C}^{\infty}\left(\mathbb{R}^{n \times m}\right)$ the class of smooth functions $f: \mathbb{R}^{n \times m} \rightarrow \mathbb{R}$ and by $\mathcal{F}=\mathcal{F}\left(\mathbb{R}^{n \times m}\right)$ the class of all extended real-valued functions $f: \mathbb{R}^{n \times m} \rightarrow \mathbb{R} \cup\{ \pm \infty\}$.

Definition. Let $r>0$ and let $C: \mathcal{C}^{\infty} \rightarrow \mathcal{F}$ be a mapping. We say that $C$ is $r$-local if for all $f, g \in \mathcal{C}^{\infty}$ and all $X_{0} \in \mathbb{R}^{n \times m}$ we have that if $f(X)=g(X)$ for $\left|X-X_{0}\right|<r$, then

$$
C(f)\left(X_{0}\right)=C(g)\left(X_{0}\right) .
$$

Notice that if we define the mapping $C_{r c}: \mathcal{C}^{\infty} \rightarrow \mathcal{F}$ as

$$
C_{r c}(f)(X)=\inf \left\{D^{2} f(X)(a \otimes b, a \otimes b): a \in \mathbb{R}^{n}, b \in \mathbb{R}^{m}\right\}, X \in \mathbb{R}^{n \times m},
$$

then $f$ is rank-1 convex if and only if $C_{r c}(f) \equiv 0$. Furthermore, the mapping $C_{r c}$ is $r$-local for any $r>0$.

Corollary 2. Let $n, m \geq 2$ and $r>0$. There does not exist an $r$-local mapping $C: \mathcal{C}^{\infty} \rightarrow \mathcal{F}$ with the property that $f$ is polyconvex if and only if $C(f) \equiv 0$.

The proofs of these results are based on a similar idea used in [5]. This is reminiscent of an idea used by Tartar in [14].

The polyconvex envelope, $f^{p c}$, of a function $f: \mathbb{R}^{2 \times 2} \rightarrow \mathbb{R}$ is by definition the extended real-valued function defined as

$$
f^{p c}(X)=\sup \{g(X): g \text { polyconvex, } g \leq f\} .
$$

It is not hard to show that either $f^{p c} \equiv-\infty$ or $f^{p c}$ is everywhere finite and polyconvex. We make use of the following two results.

Proposition 3. Let $f: \mathbb{R}^{2 \times 2} \rightarrow \mathbb{R}$ be a function and let $f^{p c}$ denote the polyconvex envelope of $f$. Then

$$
f^{p c}(X)=\inf \left\{\sum_{j=1}^{6} t_{j} f\left(X_{j}\right): t_{j} \geq 0, \sum_{j=1}^{6} t_{j}=1,(X, \operatorname{det} X)=\sum_{j=1}^{6} t_{j}\left(X_{j}, \operatorname{det} X_{j}\right)\right\}
$$

for $X \in \mathbb{R}^{2 \times 2}$.

Remark. We do not exclude the case where $f^{p c} \equiv-\infty$. The proof can be found in [6]; it can also be inferred from [4], Chap. 5.

Proposition 4 (Terpstra [15], Serre [11], Marcellini 8]). Let $f: \mathbb{R}^{2 \times 2} \rightarrow \mathbb{R}$ be a rank-1 convex quadratic form. Then $f$ is polyconvex, and therefore

$$
f(X)=q(X)+\alpha \operatorname{det} X
$$

where $q$ is a positively semi-definite quadratic form and $\alpha \in \mathbb{R}$.

Remark. If $f$ satisfies a strict Legendre-Hadamard condition, i.e. there exists a positive number $c>0$ such that

$$
f(a \otimes b) \geq c|a|^{2}|b|^{2}
$$

for all $a, b \in \mathbb{R}^{2}$, then the quadratic form $q$ in the decomposition (1) can be taken to be positive definite (cf. [8] ). 
For a $\mathcal{C}^{2}$-function $f: \mathbb{R}^{2 \times 2} \rightarrow \mathbb{R}$ we have by Taylor's formula

$$
f(X+Y)=f(X)+D f(X) Y+\frac{1}{2} D^{2} f(X)(Y ; Y)+R(X ; Y),
$$

where the remainder term $R(X ; Y)$ is given by

$$
R(X ; Y)=\int_{0}^{1}(1-t)\left(D^{2} f(X+t Y)-D^{2} f(X)\right)(Y ; Y) d t .
$$

For notational reasons it is convenient to introduce an auxiliary function, which essentially is a continuity modulus for the second derivative of $f$. For each $r \in$ $(0, \infty)$ define $\Omega_{r}:(0, \infty) \rightarrow[0, \infty)$ as (the norm being the usual one for bilinear mappings)

$$
\Omega_{r}(t)=\sup \left\{\left|D^{2} f(X+Y)-D^{2} f(X)\right|:|X| \leq r,|Y|<t\right\} .
$$

Obviously, $\Omega_{r}$ is non-decreasing and continuous, and since $D^{2} f$ is uniformly continuous on compact sets, $\Omega_{r}(t) \rightarrow 0$ as $t \rightarrow 0^{+}$. Furthermore we notice that if $|X| \leq r$, then

$$
|R(X ; Y)| \leq \frac{1}{2} \Omega_{r}(|Y|)|Y|^{2}
$$

for all $Y \in \mathbb{R}^{2 \times 2}$.

Lemma 5. Let $f: \mathbb{R}^{2 \times 2} \rightarrow \mathbb{R}$ be a $\mathcal{C}^{2}$-function such that $D^{2} f(X)(a \otimes b, a \otimes b) \geq 0$ for all $a, b \in \mathbb{R}^{2}$ and $|X|<r$. For $\varepsilon>0$ we let $\delta=(1 / 2) \sup \left\{t \in(0, r): \varepsilon \geq \Omega_{r}(t)\right\}$. Then there exists a polyconvex function $g: \mathbb{R}^{2 \times 2} \rightarrow \mathbb{R}$ such that

$$
f(X)+\varepsilon|X|^{2}=g(X) \text { if }|X| \leq \delta .
$$

Remark. An example of a quasiconvex homogeneous quartic $p: \mathbb{R}^{2 \times 2} \rightarrow \mathbb{R}$, which is not polyconvex, can be found in [1. It is clear that $p$ cannot be equal to a polyconvex function in any neighbourhood of 0 . Consequently, the term ' $\varepsilon|X|^{2}$, cannot be omitted in (3).

Proof. Put $f_{\varepsilon}(X)=f(X)+\varepsilon|X|^{2}$ and define the function $g=G^{p c}$, where $G(X)=$ $f_{\varepsilon}(X)$ if $|X| \leq \delta$ and

$$
G(X)=\sup _{|Y| \leq \delta}\left(f_{\varepsilon}(Y)+D f_{\varepsilon}(Y)(X-Y)+\frac{1}{2} D^{2} f_{\varepsilon}(Y)(X-Y, X-Y)\right)
$$

if $|X|>\delta$. Then obviously $g$ is polyconvex and $g(X) \leq f_{\varepsilon}(X)$ for $|X| \leq \delta$. We claim that $g(X)=f_{\varepsilon}(X)$ for $|X| \leq \delta$. Fix $X$ with $|X|<\delta$ and let $\sigma>0$. By Proposition 3 we can find a convex combination $\sum_{j=1}^{n} t_{j} X_{j}$ of $X$ with $\operatorname{det} X=\sum_{j=1}^{n} t_{j} \operatorname{det} X_{j}$ such that

$$
g(X)+\sigma>\sum_{j=1}^{n} t_{j} G\left(X_{j}\right)
$$

From the definition of $G$ we deduce

$$
\begin{gathered}
g(X)+\sigma>\sum_{\left|X_{j}\right| \leq \delta} t_{j} f_{\varepsilon}\left(X_{j}\right) \\
+\sum_{\left|X_{j}\right|>\delta} t_{j}\left(f_{\varepsilon}(X)+D f_{\varepsilon}(X)\left(X_{j}-X\right)+\frac{1}{2} D^{2} f_{\varepsilon}(X)\left(X_{j}-X, X_{j}-X\right)\right) .
\end{gathered}
$$


If we apply Taylor's formula we see that the last sum equals

$$
\begin{gathered}
\sum_{\left|X_{j}\right| \leq \delta} t_{j} R\left(X, X_{j}-X\right) \\
+\sum_{j=1}^{n} t_{j}\left(f(X)+D f_{\varepsilon}(X)\left(X_{j}-X\right)+\frac{1}{2} D^{2} f_{\varepsilon}(X)\left(X_{j}-X, X_{j}-X\right)\right)
\end{gathered}
$$

and estimating this sum from below using (2) and the choice of $\delta$ we get

$$
g(X)+\sigma>f_{\varepsilon}(X)+\frac{1}{2} \sum_{\left|X_{j}\right| \leq \delta} t_{j}\left|X_{j}\right|^{2}\left(\varepsilon-\Omega_{r}\left(\left|X_{j}-X\right|\right)\right) d x \geq f_{\varepsilon}(X) .
$$

This concludes the proof.

Proof of Theorem 1. It suffices to prove the first assertion for the case $m=n=2$. Let $h: \mathbb{R}^{2 \times 2} \rightarrow \mathbb{R}$ be a smooth function which is rank-1 convex, but not polyconvex (see, e.g., [2, [1] or [12]). Take for each $s>1$ two auxiliary functions $\zeta_{s}, \xi_{s} \in \mathcal{C}^{\infty}(\mathbb{R})$ verifying

$$
\begin{gathered}
\zeta_{s}(t)= \begin{cases}1 & \text { if } t<s, \\
0 & \text { if } t>s+1,\end{cases} \\
\xi_{s}(t)= \begin{cases}0 & \text { if } t<s-1, \\
t^{2} & \text { if } t>s+1,\end{cases}
\end{gathered}
$$

and $\xi_{s}$ non-decreasing, convex and $\xi_{s}^{\prime \prime}(t)>0$ for $t \in(s-1, s+1)$. It is not difficult to see that there exist $s>1$ and $k>0$ such that

$$
h(X) \zeta_{s}(|X|)+k \xi_{s}(|X|)
$$

is rank-1 convex, but not polyconvex. Further choose $\varepsilon>0$ such that

$$
g(X)=h(X) \zeta_{s}(|X|)+k \xi_{s}(|X|)+\varepsilon|X|^{2}
$$

is not polyconvex. If $R(X, Y)$ denotes the remainder term in the Taylor expansion of $g$ about $X$, then there exists a constant $C>0$ such that

$$
|R(X, Y)| \leq 3 \int_{0}^{1}(1-t)^{2} \sum_{|\alpha|=3}\left|\partial^{\alpha} g(X+t Y) \frac{Y^{\alpha}}{\alpha !}\right| d t \leq C|Y|^{3}
$$

for all $X, Y \in \mathbb{R}^{2 \times 2}$. In the notation of Lemma [5] (see (2) this corresponds to $\Omega_{r}(t) \leq 2 C t, t>0$, independently of $r>0$.

Fix $X_{0} \in \mathbb{R}^{2 \times 2}$. We claim that there exists a polyconvex extension of $g$ from the closed ball $\left|X-X_{0}\right| \leq \varepsilon /(4 C)$. Indeed, define $g_{X_{0}}(X)=g\left(X_{0}+X\right)$ and notice that by Lemma 5 we may find a polyconvex function $G_{X_{0}}$ such that $g\left(X+X_{0}\right)=$ $g_{X_{0}}(X)=G_{X_{0}}(X)$ for $|X| \leq \varepsilon /(4 C)$, or equivalently, such that

$$
g(X)=G_{X_{0}}\left(X-X_{0}\right) \quad \text { for } \quad\left|X-X_{0}\right| \leq \frac{\varepsilon}{4 C}
$$

which proves the claim. Finally, we define the function $f$ as

$$
f(X)=g\left(\frac{4 C}{\varepsilon} X\right), X \in \mathbb{R}^{n \times m}
$$

To obtain a function with smooth polyconvex extensions we mollify $f$ using a nonnegative mollifier. This finishes the proof of the first assertion. To prove the second assertion we notice that according to Šverák [13] there exists a polynomial $p$ of 
degree four on $\mathbb{R}^{3 \times 2}$, which is rank-1 convex but not quasiconvex. We may now repeat the above construction with $h=p$ to obtain the conclusion. However, instead of using Proposition 4 we apply a more general result of Terpstra [15] (see also Serre [11]). This result states that for $\min \{m, n\}=2$ and for quadratic forms on $\mathbb{R}^{n \times m}$, rank-1 convexity is equivalent to polyconvexity. We also need to use a higher dimensional variant of Proposition 3 that can be deduced from [4, Chap. 5 (see also [6]).

\section{ACKNOWLEDGEMENTS}

I would like to thank Bernd Kirchheim for stimulating discussions related to the subject of the paper. The work was finished while the author was visiting the Max-Planck Institute for Mathematics in the Sciences, Leipzig. The support from the Danish Natural Science Research Council and from the MPI is gratefully acknowledged.

\section{REFERENCES}

[1] J.J. Alibert, B. Dacorogna. An example of a quasiconvex function not polyconvex in dimension two. Arch. Rat. Mech. Anal. 117: 155-66, 1992. [MR 92k:49019]

[2] G. Aubert. On a counterexample of a rank 1 convex function which is not polyconvex in the case $N=2$. Proc. Roy. Soc. Edinburgh Sect. A 106: 237-40, 1987. MR 89f:26016

[3] J.M. Ball. Convexity conditions and existence theorems in nonlinear elasticity. Arch. Rat. Mech. Anal. 63: 337-403, 1978. MR 57:14788

[4] B. Dacorogna. Direct Methods in the Calculus of Variations. Springer-Verlag Berlin/Heidelberg 1989. MR 90e:49001

[5] J. Kristensen. On the non-locality of quasiconvexity. I.H.P. Analyse Non Linéaire, to appear.

[6] J. Kristensen. On quasiconvex envelopes of locally bounded functions. Preprint 1996.

[7] T. Iwaniec, A. Lutoborski. Polyconvex functionals for nearly conformal deformations. SIAM J. Math. Anal. 27, 3: 609-19, 1996. MR 97d:49004

[8] P. Marcellini. Quasiconvex quadratic forms in two dimensions. Appl. Math. Optimization 11: 183-89, 1984. MR 85j:26018

[9] C.B. Morrey. Quasiconvexity and the semicontinuity of multiple integrals. Pacific J. Math. 2: 25-53, 1952. MR 14:992a

[10] S. Müller. Variational models for microstructure and phase transitions. Lectures at the C.I.M.E. summer school 'Calculus of variations and geometric evolution problems', Cetraro 1996.

[11] D. Serre. Formes quadratiques et calcul des variations. J. Math. pures et appl. 62: 177-196, 1983. MR 84m:49031

[12] V. Šverák. Quasiconvex functions with subquadratic growth. Proc. Roy. Soc. London 433: 723-25, 1991. MR 92f:26022

[13] V. Šverák. Rank-one convexity does not imply quasiconvexity. Proc. Roy. Soc. Edinburgh 120A: 185-89, 1992. MR 93b:49026

[14] L. Tartar. The compensated compactness method applied to systems of conservation laws. In "Systems of Nonlinear Partial Differential Equations"; J.M. Ball (ed.): 263-85. (D.Reidel Publ. Company), 1983. MR 85e:35079

[15] F.J. Terpstra. Die darstellung der biquadratischen formen als summen von quadraten mit anwendung auf die variationsrechnung. Math. Ann. 116: 166-80, 1938.

Mathematical Institute, University of Oxford, 24-29 St Giles, Oxford OX1 3LB, UNITED KINGDOM

E-mail address: kristens@maths.ox.ac.uk 